\title{
Automated Total IntraVenous Anesthesia (amTIVA) from induction to recovery
}

\author{
Luis A. Paz*, Margarida M. Silva* ${ }^{*} \ddagger$, Simão Esteves ${ }^{\S}$, Rui Rabiço ${ }^{\text {I }}$, Teresa Mendonça * $\ddagger$ \\ *Dep. Matemática, Fac. Ciências, Universidade do Porto, Porto, Portugal: lpaz@fe.up.pt; tmendo@fc.up.pt \\ $\dagger^{\dagger}$ Dep. Information Technology, Uppsala University, Uppsala, Sweden: margarida.silva @ fc.up.pt \\ ${ }^{\ddagger}$ Center for Research and Development in Mathematics and Applications, Universidade de Aveiro, Aveiro, Portugal

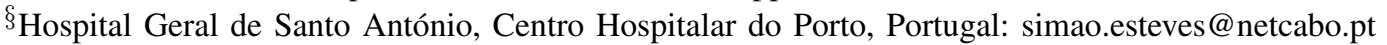

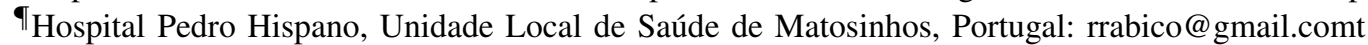

\begin{abstract}
This paper presents a multi-functional automatic control approach for total intravenous anesthesia (TIVA) administration, valid for several drug combinations and able to run autonomously during the three main phases of general anesthesia: induction, maintenance and recovery. While using this standalone TIVA module, named as amTIVA, the anesthesiologist has the crucial role of system supervisor. Bolus administration, openloop target controlled infusion (TCI) and closed-loop controlled infusion modes are implemented and available for the control of the two main components of anesthesia: the neuromuscular blockade and the depth of anesthesia. This multi-functional module is implemented in the GALENO Platform and was successfully tested in more than thirty clinical cases.
\end{abstract}

\section{INTRODUCTION}

Total intravenous anesthesia (TIVA) is a technique of general anesthesia where all the anesthetic drugs are intravenously administered. During the induction phase the patient is brought to a reversible state of hypnosis, analgesia and muscle relaxation through the administration of hypnotics, analgesics and muscle relaxants. After induction, during the maintenance phase, the patient must remain in the anesthetic condition adequate for the surgery. The recovery phase occurs after the cessation of administration of the intravenous drugs and enables the return to baseline physiologic functions.

Mainly driven by the potential of automation in several applications [1], the subject of automation in anesthesia has focused the efforts of many research teams worldwide during the last decades. Despite the great progress that has occurred, the day when the anesthesiologist is replaced completely by a machine is still unforeseeable. In spite of the several sophisticated devices that exist nowadays in the operating room, the anesthesiologist is still the sole player in the process of defining the amount of drugs to be administered to the patients so that the physiologic variables that are measured from the patient follow the pre-defined references or lay inside the admissible physiologic ranges.

This paper presents a multi-functional automatic control approach for TIVA administration, named as amTIVA, in which the anesthesiologist becomes supervisor of the whole process. This constitutes the first contribution of the paper. The whole strategy is implemented within the GALENO Platform [2] and incorporates several controllers for the two main components of anesthesia: the neuromuscular blockade (NMB) and the



Fig. 1: Structure of the multi-functional automatic controller amTIVA as implemented in the GALENO Platform.

depth of anesthesia (DoA). A schematic representation of the whole system is shown in Fig. 1. The second contribution of the paper is the development of a novel and heuristic approach for Target Controlled Infusion (TCI) for the DoA, hereafter referred as GALENO-TCI. The third contribution of the paper is the assessment of the performance of the amTIVA for the control of the NMB and the DoA in more than thirty real cases of general anesthesia.

The remainder of this paper is organized as follows. Section II describes the system architecture and the basic concepts behind the NMB and BIS measurements. Section III presents three control algorithms for the induction and maintenance of anesthesia. Section IV shows the results obtained when the full control strategy was used in the clinical setting and section $\mathrm{V}$ draws the conclusions.

\section{SYSTEM SETUP: SENSORS AND MODULE ARCHITECTURE}

\section{A. NMB and BIS measurements}

A commonly used method to monitor the NMB is by using of the first response (T1\%) of a Train-of-Four (TOF) electrical 
stimulation of the ulnar nerve at the patient's hand calibrated by a reference twitch [3].

The hypnotic component of the DoA can be evaluated by the bispectral index (BIS) [4], a multi-factorial parameter derived from the electroencephalogram. The BIS is a dimensionless number that varies from 0 (corresponding to total suppression of cortical electrical activity) to 97.7 (corresponding to full cortical electrical activity). A BIS between 40 and 60 is associated with a low probability of intra-operative awakening and awareness, and is recommended for general anesthesia.

The appropriate measurement of the TOF and BIS responses is a requisite for the proper performance of the whole control strategy. Hence, the specifications for placement of the electrodes and sensors as established by manufacturers should be strictly followed.

\section{B. Automated module for TIVA (amTIVA)}

Figure 2 shows the graphic user interface of the amTIVA module developed for the automated TIVA administration. The flowchart of the amTIVA was inspired by the work in [5] and was further extended to the induction phase. The amTIVA works at a higher level by managing the NMB control module and the DoA/BIS control module, and it incorporates the followings features to facilitate the sequentiality between the three anesthesia phases, thereby increasing its practical clinical performance:

1) The anesthesiologist configures the amTIVA based on the anesthetic protocol, setting the adequate drugs: analgesic (remifentanil, sufentanil or fentanil), hypnotic (propofol) and muscle relaxant (rocuronium or atracurium).

2) The anesthesiologist selects, through the amTIVA interface, the following types of analgesic and hypnotic drugs administration: i) target controlled infusion (TCI), ii) bolus in $\mu / \mathrm{kg}$ or iii) pre-defined continued infusion rate in $m l / h$. It should be noted that constraints were added to the interface so that fentanil is only manually delivered. Moreover, due to clinical constraints during the induction phase the muscle relaxant can only be administered by bolus.

The amTIVA also includes:

- A button to manual clinical validation of the NMB sensor calibration ("NMB S.Cali-OKt't').

- A button indicating the completion of the intubation and the end of the induction phase ('Intubation-OKt' $t$ ').

\section{CONTROL STRATEGIES}

\section{A. Induction phase}

The induction phase of general anesthesia is characterized by the administration of high doses of anesthetics to the patients, aiming at reaching a reversible state of hypnosis, analgesia, and muscle relaxation in a short time. The recommended amount of each anesthetic drug for induction follows population studies and is well defined for each clinical case. Since it is very difficult to predict accurately the pharmacokinetic (PK)

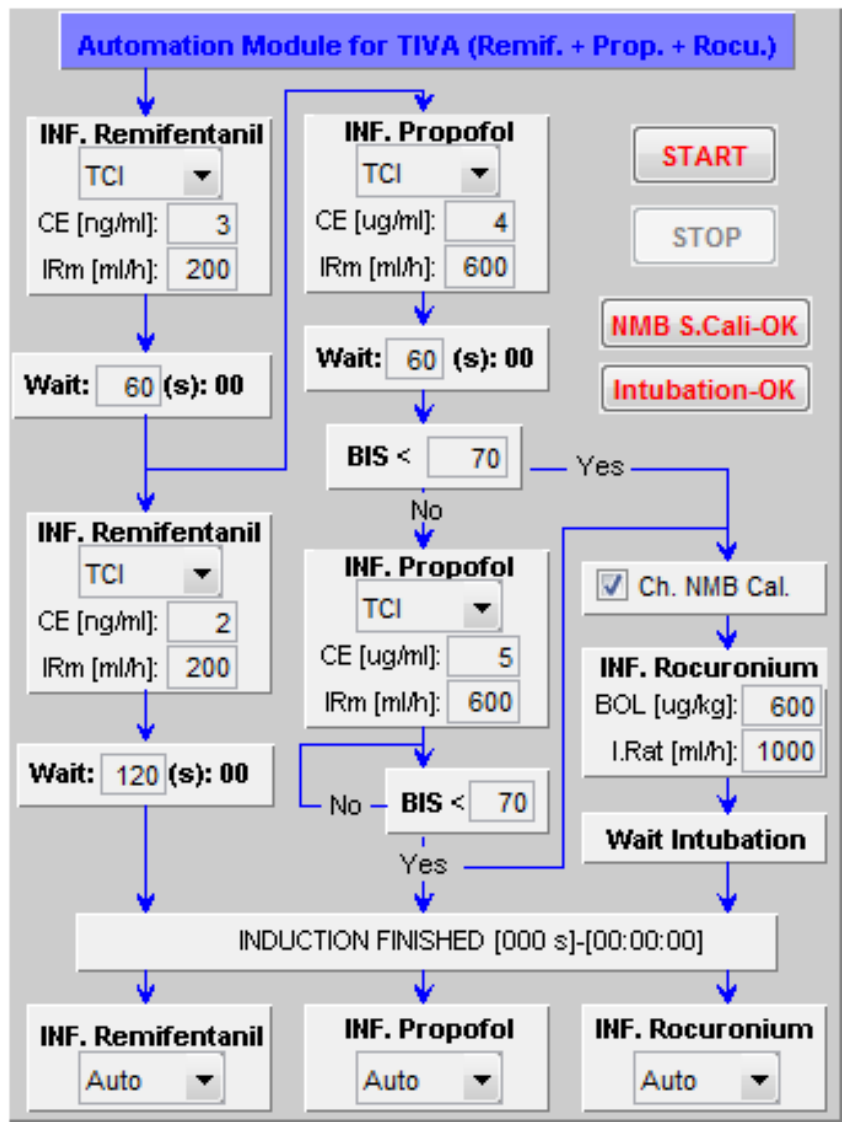

Fig. 2: GUI of the automated control module for TIVA (amTIVA). The combination of remifentanil, propofol and rocuronium is here used as an example.

and pharmacodynamic (PD) response of each patient to these predefined population doses, the amTIVA is defined such that, during the induction phase and until the patient's physiologic signals had stabilized, the anesthetic drugs are administered by bolus or by open-loop controllers as TCI, instead of by closed-loop controllers.

1) Neuromuscular blockade: Due to clinical restrictions the induction of the NMB is always performed through an initial bolus administration. Even though being automatically administered by the syringe pumps, the amount and infusion rate of the muscle relaxant drug has to be defined in the "INF. Rocuronium 't' block in Fig. 2.

2) Depth of anesthesia: The induction phase of DoA may be performed through different protocols as explained in section II-B. In this subsection the novel and heuristic approach GALENO-TCI that is proposed in this paper is described.

The main idea of TCI is to use a predefined population $\mathrm{PK} / \mathrm{PD}$ model to determine the plasma or/and effect site concentration, and to compute the amount of drug needed to reach the target concentration by "inversion" of the model. The GALENO-TCI is a heuristic predictive TCI algorithm that uses the compartment PK/PD models in [6], [7], [8], [9] 




Fig. 3: Representation of parametric tri-compartmental PK model plus the effect compartment.

for propofol, remifentanil and sufentanil, as predictive models. Figure 3 shows a general scheme of these tri-compartmental models, being its mathematical representation [10] given by

$$
\begin{gathered}
\frac{d C_{1}}{d t}=\frac{I R-\left(k_{10}+k_{12}+k_{13}\right) V_{1} C_{1}+k_{21} V_{2} C_{2}+k_{31} V_{3} C_{3}}{V_{1}} \\
\frac{d C_{2}}{d t}=\frac{k_{12} V_{1} C_{1}-k_{21} V_{2} C_{2}}{V_{2}} \\
\frac{d C_{3}}{d t}=\frac{k_{13} V_{1} C_{1}-k_{31} V_{3} C_{3}}{V_{3}} \\
\frac{d C E}{d t}=k_{e 0} C_{1}-k_{e 0} C E
\end{gathered}
$$

where:

$k_{i j}$ is the transfer rate from compartment $i$ to $j\left[\mathrm{~min}^{-1}\right]$; $V_{i}$ is the volume of the compartment $i[\mathrm{ml}]$;

$C_{1}$ is the plasma concentration $\left[Q D^{1} / \mathrm{ml}\right]$;

$C_{2}, C_{3}$ are the peripheral compartment concentrations $[Q D / m l]$;

$C E$ is the effect site concentration $[Q D / m l]$;

$I R$ is the drug infusion rate $[Q D / \min ]$.

For the digital implementation of the GALENO-TCI, the continuous time equations (1) were transformed to discrete time (2) using the zero-order hold method (see e.g. [11]) and sampling time $T_{s}$ as

$$
\begin{gathered}
C_{1}[n+1]=\left(\frac{I R[n]-\left(k_{10}+k_{12}+k_{13}\right) V_{1} C_{1}[n]}{V_{1}}\right) T_{s}+ \\
\left(\frac{k_{21} V_{2} C_{2}[n-1]+k_{31} V_{3} C_{3}[n]}{V_{1}}\right) T_{s}+C_{1}[n] \\
C_{2}[n+1]=\left(\frac{k_{12} V_{1} C_{1}[n]-k_{21} V_{2} C_{2}[n]}{V_{2}}\right) T_{s}+C_{2}[n] \\
C_{3}[n+1]=\left(\frac{k_{13} V_{1} C_{1}[n]-k_{31} V_{3} C_{3}[n]}{V_{3}}\right) T_{s}+C_{3}[n] \\
C E[n+1]=\left(k_{e 0} C_{1}[n]-k_{e 0} C E[n]\right) T_{s}+C E[n]
\end{gathered}
$$

When the target of the TCI strategy is the plasma concentration $C_{1}$, the controller determines the value $I R[n]$ that

\footnotetext{
${ }^{1} Q D$ is the notation to represent the quantity of drug $(n g, \mu g, m g)$, this must be the same for the compartment concentration that for the infusion rate
}

matches $C_{1}$ with the reference or target concentration $C T$ and the solution is obtained by substituting (3) in (2a), and solving for $I R[n]$ as

$$
\begin{gathered}
C_{1}[n+1]=C T \\
I R[n]=\frac{\left(C T-C_{1}[n]\right) V_{1}}{T_{s}}+\left(k_{10}+k_{12}+k_{13}\right) V_{1} C_{1}[n] \\
-k_{21} V_{2} C_{2}[n]-k_{31} V_{3} C_{3}[n]
\end{gathered}
$$

where (4) is subject to

$$
0 \leq I R[n] \leq I R_{\max }
$$

When the target of the TCI strategy is the effect site concentration $C E$, the problem increases in complexity. The controller must determine the value of $I R[n]$ that matches $C_{1}, C E$ and $C T$ (6). The first step to obtain the solution is by substituting (6) and (2a) in (2d) and solving for $I R[n]$ as

$$
C E[n+1]=C_{1}[n+1]=C T
$$

$$
\begin{aligned}
& I R[n]=V 1\left(\frac{\left.\frac{C T-C E[n]}{k_{e 0} T_{s}}+C E[n]-C_{1}[n]\right)}{T_{s}}\right)+ \\
& \left(k_{10}+k_{12}+k_{13}\right) V_{1} C_{1}[n]-k_{21} V_{2} C_{2}[n]-k_{31} V_{3} C_{3}[n]
\end{aligned}
$$

where (7) is subject to the constraints (5) and

$$
\begin{aligned}
& C E[n+1]=C_{1}[n+1]=C T \\
& C E[n+2]=C_{1}[n+2]=C T
\end{aligned}
$$

The value $I R[n]$ that is solution of the effect site TCI problem depends on the current process status $C_{1}[n], C_{2}[n], C_{3}[n]$ and $C E[n]$. The flowchart of the solution proposed for the effect site TCI problem (GALENO-TCI algorithm) is presented in Fig. 4. This algorithm is used at each sampling time and its main steps are summarized below:

1) Determine the solution $I R[n]$ using (7);

2) Calculate the predictive $C_{1}[n+1]$ and $C E[n+1]$, using (2);

3) Calculate the predictive $C_{1}[n+2]$ and $C E[n+2]$, assuming $\operatorname{IR}[n+1]=0$, using (2);

4) $I R[n]$ is considered a valid input, satisfying (8), if $C_{1}[n+2] \leq C E[n+2]$

5) Otherwise (if $C_{1}[n+2]>C E[n+2]$ ), re-calculate $I R[n]$ using the recursive iterative method described below:

Numerical iterative method: This iterative procedure for calculating $\operatorname{IR}[n]$ is only used in the case of $C_{1}[n+2]>$ $C E[n+2]$, meaning that the problem not be solved in one sampling time. This fact is not a drawback, since the constraints (8) may be reformulated by introducing $p$ sampling time, where $p=1,2,3, \ldots$, as

$$
\begin{gathered}
C E[n+p]=C_{1}[n+p]=C T \\
C E[n+p+1]=C_{1}[n+p+1]=C T
\end{gathered}
$$

This procedure searches for a solution satisfying (9) and minimizing $p$. It is based in the iterative method represented 


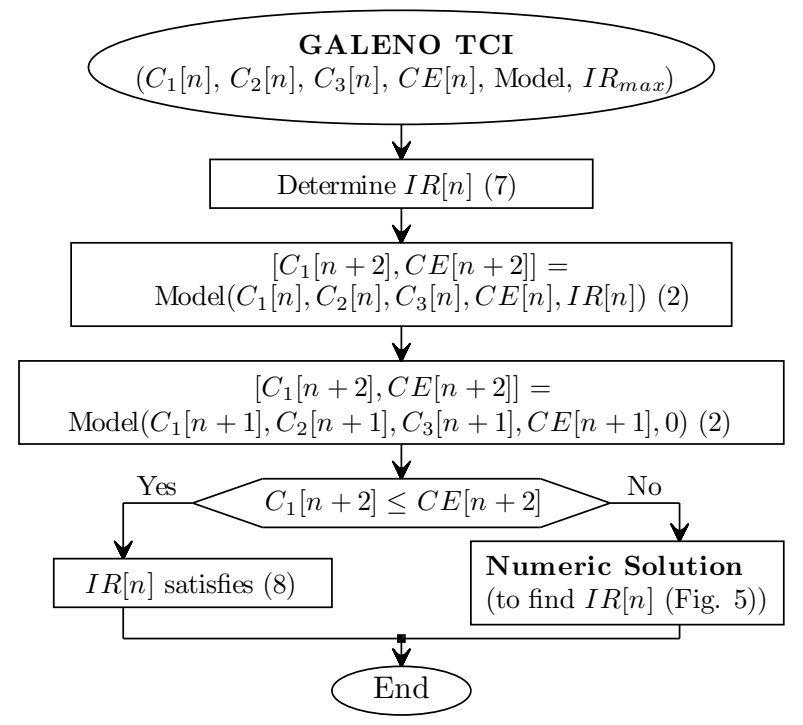

Fig. 4: Flowchart of GALENO-TCI for effect site concentration.

in Fig. 5. This procedure is a practical and straightforward criterion based on the hardware constrains, e.g. the ALARIS GH syringe pump has infusion rate range between 0 and 1200 $\mathrm{ml} / \mathrm{h}$, with increment of $0.1 \mathrm{ml} / \mathrm{h}$; thus after 15 iterations $1200 / 2^{15}=0.036<0.1$ is obtained. Figure 6 shows the performance of the GALENO-TCI for propofol using the Schnider's model [7]. The upper plot of Fig. 6 shows the effect site concentration $\mathrm{CE}$, in thick solid line, reaching the target effect site concentration CT, represented by the line with dots, as desired. The concentration of the drug (propofol) in the plasma is represented by $\mathrm{C} 1$, in thin solid line. This regulation is due to the administered infusion IR, in the bottom plot of Fig. 6.

\section{B. Maintenance phase}

The practical experience achieved in the GALENO project [12] over 350 clinical trials suggest that the ideal time to enable the automatic controller for the NMB is after the spontaneous recovery of NMB after the initial bolus. This time instant is usually determined by the "OnLine tuned Algorithm for Recovery Detection" [13]. Similarly, experience suggests that the ideal time to enable the automatic controllers for the DoA is after completion of the induction phase, when the BIS reaches a value that is close to the desired reference.

1) Control of neuromuscular blockade: One of the controllers that are implemented in the GALENO Platform for the control of the NMB after recovery from the initial bolus is a self-calibrating total-mass controller for the NMB based on the minimally parameterized parsimonious Wiener model described in detail in [14]. The idea behind it is that, by the use of the parameters of the model [15] describing the effect of muscle relaxants in the NMB that are on-line identified by an extended Kalman Filter (EKF), the total mass of muscle relaxant in the different compartments of the human body

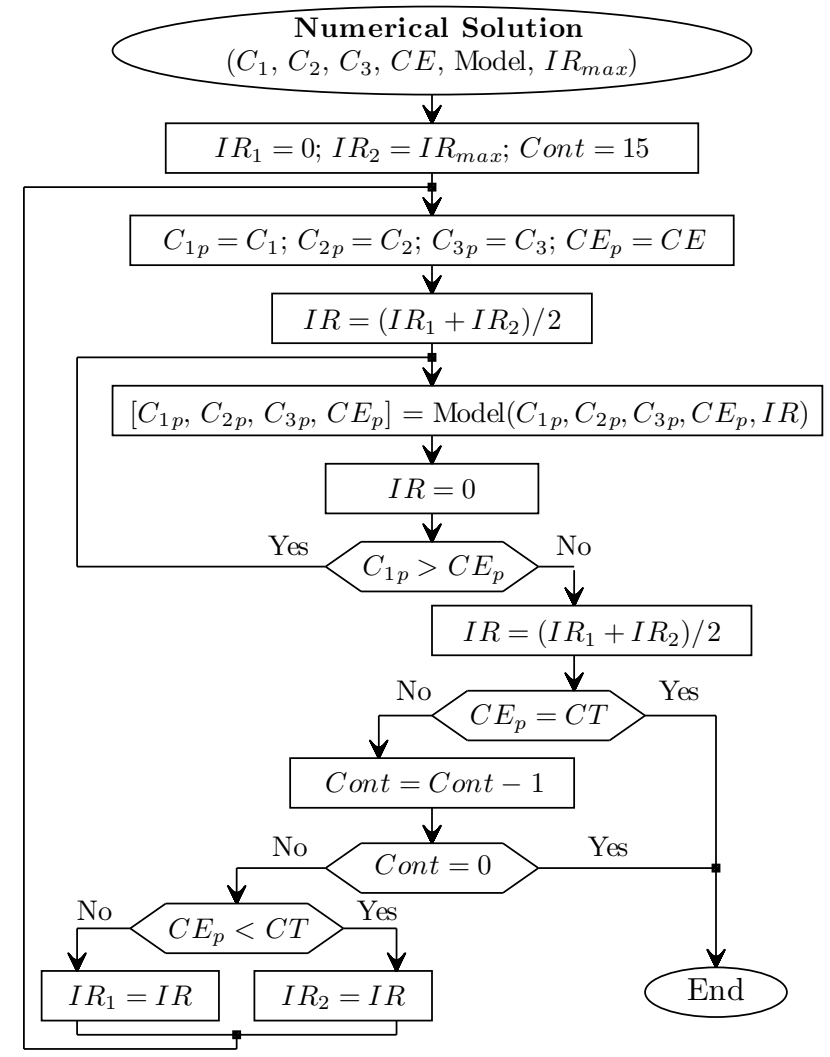

Fig. 5: Flowchart of successive approximations method used in GALENO-TCI.
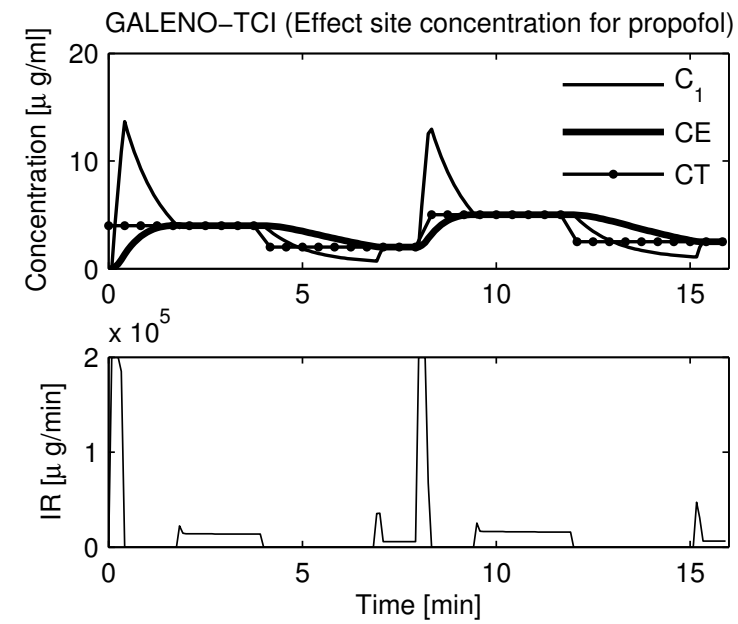

Fig. 6: Performance of GALENO-TCI for propofol administration using Schnider's model. CT is the target effect site concentration, $\mathrm{C} 1$ is the plasma concentration and $\mathrm{CE}$ is the effect concentration. 
can be controlled [16] so that the measured TOF T1 response follows a predefined set-point (usually 5-10\%).

2) Control of depth of anesthesia: The scheduled strategy to control the DoA uses the hypnotic and the analgesic drug as control variables. The hypnotic drug administration (propofol) is controlled with a cascade of a closed-loop GALENO-TCI and a proportional integral (PI) controller [2]. The analgesic drugs administration (remifentanil or sufentanil) is controlled with the open-loop GALENO-TCI.

The premise used to develop the closed-loop GALENOTCI-PI is based on the assumption that in a real patient, 2.5 min after a step change on the TCI reference, the drug concentration at the effect site $C E[n]$ reaches steady state. The GALENO-TCI block has a fixed sampling time $\left(T_{s_{T C I}}\right)$ of 5 seconds and calculates the infusion rate of propofol that should be given to the patient so that the effect site target concentration is reached. The PI block has a variable sampling time $\left(2.5 \leq T_{s_{P I}} \geq 5 \mathrm{~min}\right)$ and determines the effect site target concentration $(C T)$ that tracks the BIS reference $\left(B I S_{\text {ref }}\right)$ using

$$
C T[n+1]=C T[n]+\frac{1}{k_{p}}\left(B I S_{r e f}-B I S[n]\right),
$$

where $k_{p}$ is a proportional gain that was empirically assumed as equal to $k_{p}=-10$.

\section{Results}

The amTIVA described in section II-B comprising the GALENO-TCI, and the NMB and the DoA controllers described in section III, was successfully validated in more than thirty patients undergoing general anesthesia.

Figures 7,8 and 9 illustrate the performance of the amTIVA for three cases with different anesthetic drug combinations: rocuronium/propofol/remifentanil, rocuronium/propofol/sufentanil and rocuronium/propofol/fentanil, respectively.

The GALENO-TCI algorithm described in section III-A2 was used during induction for both propofol, sufentanil and remifentanil, using models from [7], [8] and [9], respectively.

During maintenance of propofol administration, the GALENO-TCI-PI algorithm, described in section III-B2 was used.

For the case shown in Fig. 9 fentanil was administrated manually by bolus (see bottom plot of Fig. 9).

In all clinical trials the controlled variables (NMB and BIS) remained close to the respective references ( $N M B_{\text {ref }}=5 \%$ and $B I S_{\text {ref }}=50 \%$ ), maintaining an relative error less than $5 \%$, which is clinically accepted in anesthetic practice. The controlled variables are shown in the two upper plots of Figs. 7, 8, and 9. Moreover, the administered drug rates are in accordance with the guidelines.

\section{Conclusion}

The great challenge of complete automation of drug administration in anesthesia is overcome using the amTIVA proposed in this paper. This is shown for a variety of drugs
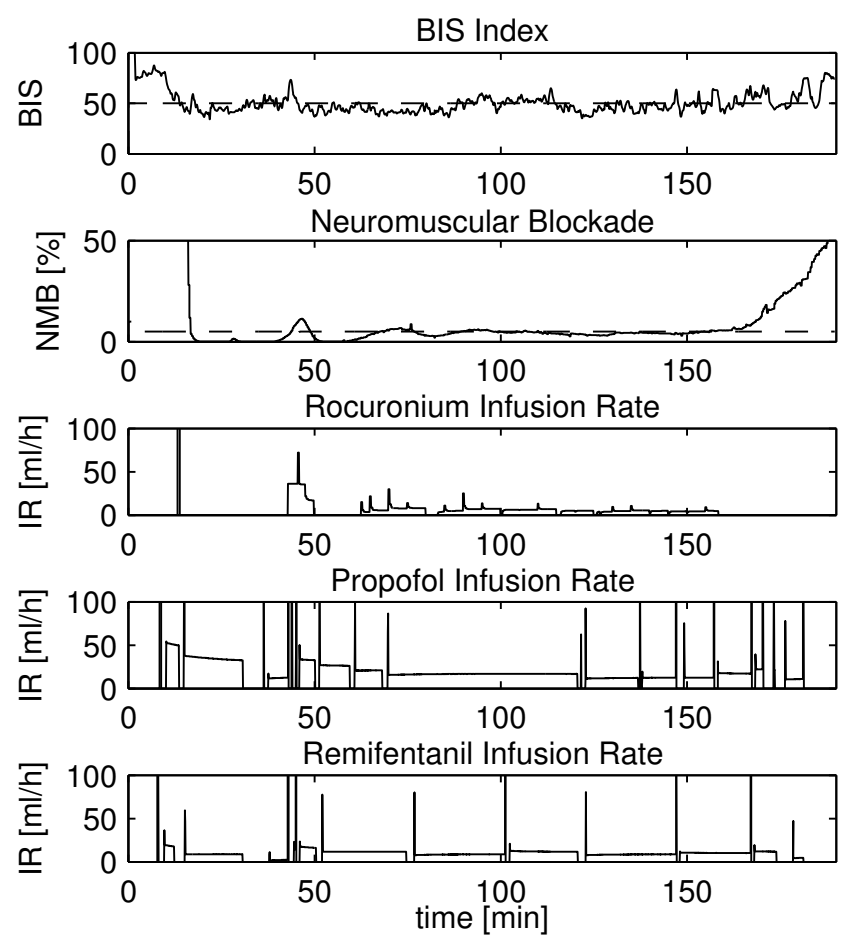

Fig. 7: Performance of the amTIVA in a real case of general anesthesia. Anesthetic drugs: rocuronium/propofol/remifentanil.
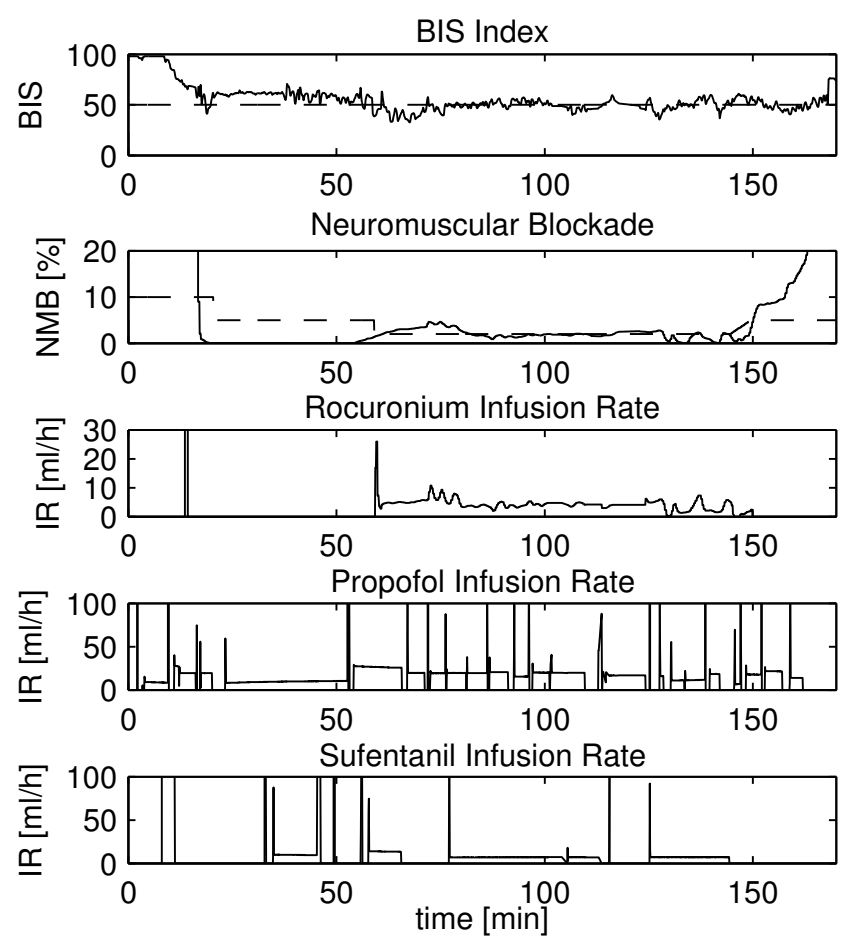

Fig. 8: Performance of the amTIVA in a real case of general anesthesia. Anesthetic drugs: rocuronium/propofol/sufentanil. 

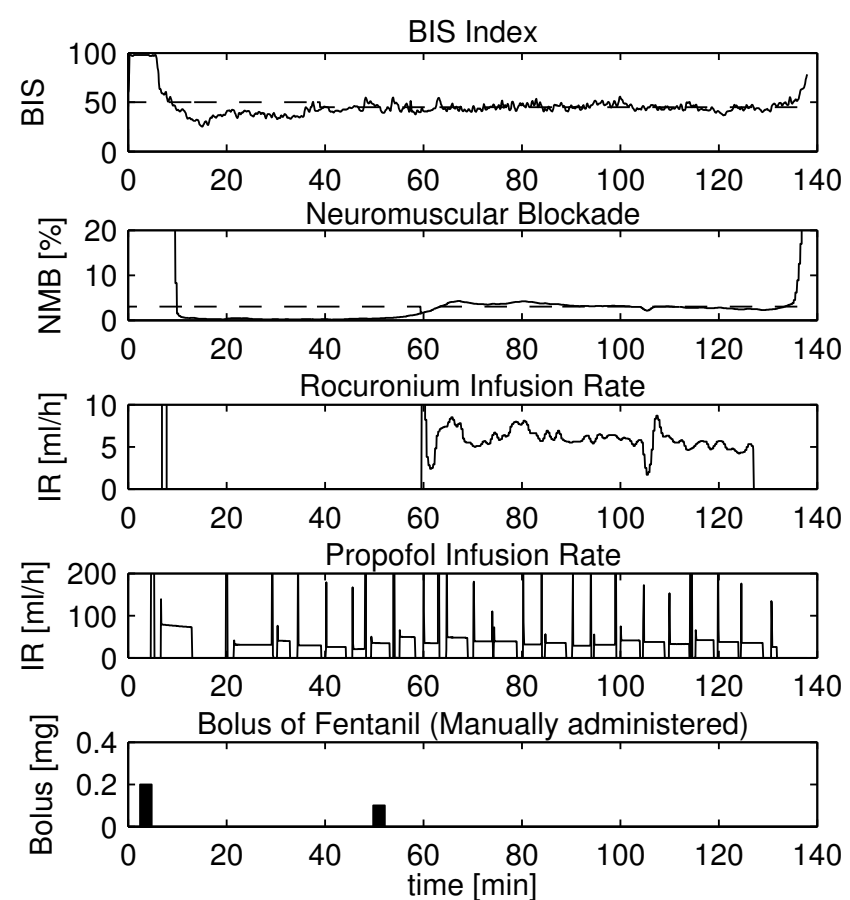

Fig. 9: Performance of the amTIVA in a real case of general anesthesia. Anesthetic drugs: rocuronium/propofol/fentanil.

and administration protocols. The cascade of a closed-loop GALENO-TCI and a PI controller for DoA with propofol [2] is able to maintain the BIS index within the clinically recommended range, even responding well to unexpected disturbances and measurement noise. At the same time, the adaptive controller based on a minimally parameterized parsimonious Wiener model for the rocuronium induced NMB achieved satisfactory tracking of the NMB reference, and the on-line identification of parameters ensures the decrease of the patient model uncertainties.

\section{ACKNOWLEDGMENTS}

This work was supported by Portuguese funds through the CIDMA - Center for Research and Development in Mathematics and Applications, and the Portuguese Foundation for Science and Technology ("FCT - Fundação para a Ciência e a Tecnologia"), within project PEst-OE/MAT/UI4106/2014 and project Galeno PTDC/SAU-BEB/103667/2008. The financial support of the European Research Council via the Advanced Grant 247035 (SysTEAM) is also to be acknowledged.

The authors would like to thank the team of anesthesiologists from the "Hospital de Santo António - Centro Hospitalar do Porto" and the "Hospital Pedro Hispano - Unidade Local de Saúde de Matosinhos" for their crucial contribution to the implementation of this project.

\section{REFERENCES}

[1] T. Samad and A. M. Annaswamy-(eds.), "The impact of control technology," IEEE Control Systems Society, 2011. [Online]. Available: www.ieeecss.org
[2] L. Paz, B. Costa, D. Caiado, S. Esteves, R. Rabiço, M. Seabra, Z. Moreira, J. M. Miranda, and T. Mendonça, "Galeno: Computer aided system for modeling, monitoring and control in anesthesia," IEEE Journal of Biomedical and Health Informatics - submitted, oct. 2013.

[3] S. Esteves, M. Seabra, M. S. Araújo, T. Mendonça, and P. Lago, "Computer controlled administration of atracurium," in Proc. EC'91 European Conference of Anesthesiology, 1991.

[4] J. C. Sigl and N. G. Chamoun, "An introduction to bispectral analysis for electoencephalogram,” J. Clinical Monitoring, vol. 10, pp. 392-404, 1994.

[5] T. M. Hemmerling, E. Arbeid, M. Wehbe, S. Cyr, R. Taddei, and C. Zaouter, "Evaluation of a novel closed-loop total intravenous anaesthesia drug delivery system: a randomized controlled trial," British Journal of Anaesthesia, vol. 110, pp. 1031Û́-1039, 2013.

[6] B. Marsh, M. White, N. Morton, and G. N. C. Kenny, "Pharmacokinetic model driven infusion of propofol in children," British Journal of Anaesthesia, vol. 67, no. 1, pp. 41-48, 1991.

[7] T. W. Schnider, C. F. Minto, P. L. Gambus, C. Andresen, D. B. Goodale, S. L. Shafer, and E. J. Youngs, "The influence of method of administration and covariates on the pharmacokinetics of propofol in adult volunteers," Anesthesiology, vol. 8, pp. 1170-1182, 1998.

[8] C. F. Minto, T. Schnider, T. Egan, E. Youngs, H. Lemmens, P. Gambus, V. Billard, J. Hoke, K. Moore, D. Hermann, K. Muir, J. Mandema, and S. Shafer, "Influence of age and gender on the pharmacokinetics and pharmacodynamics of remifentanil. I. model development," Anesthesiology, vol. 86, pp. 10-23, 1997.

[9] E. Gepts, S. Shafer, F. Camu, D. Stanski, R. Woestenborghs, A. V. Peer, and J. Heykants, "Linearity of pharmacokinetics and model estimation of sufentanil," Anesthesiology, vol. 83, no. 6, pp. 1194-1204, 1995.

[10] K. Godfrey, Compartmental models and their application. Academic Press, 1983.

[11] K. J. Åström and B. Wittenmark, Computer-Controlled Systems. Englewood Cliffs, NJ: Prentice Hall, 1984.

[12] "Project-Galeno: Modeling and control for personalized drug administration," Faculdade de Ciências, Universidade do Porto, 2008, project supported by Fundação para a Ciência e a Tecnologia de Portugal, Contract:PTDC/SAU-BEB/103667/2008. [Online]. Available: http://www2.fc.up.pt/galeno/

[13] M. M. Silva, C. Sousa, R. Sebastião, J. Gama, T. Mendonça, P. Rocha, and S. Esteves, "Total mass TCI driven by parametric estimation," in Proc. 17th Mediterranean Conference on Control and Automation (MED'09), jun. 2009, pp. 1149-1154.

[14] M. M. Silva, L. Paz, T. Wigren, and T. Mendonça, "Performance of an adaptive controller for the neuromuscular blockade based on inversion of a wiener model," Asian Journal of Control - submitted, sep. 2013.

[15] M. M. Silva, T. Wigren, and T. Mendonça, "Nonlinear identification of a minimal neuromuscular blockade model in anesthesia," IEEE Transactions on Control Systems Technology, vol. 20, no. 1, pp. 181$188,2012$.

[16] G. Bastin and A. Provost, "Feedback stabilization with positive control of dissipative compartmental systems," in Proc. 15th International Symposium on Mathematical Theory of Networks ans Systems, aug 2002. 